\title{
Ghettos am Rande der Stadt?
}

\author{
Friederike Fleischer \\ Adriana Hurtado Tarazona \\ Maria Jose Alvarez Rivadulla
}

\begin{abstract}
Konfrontiert mit kontinuierlich wachsender Nachfrage nach preiswertem Wohnraum, begann die Regierung Kolumbiens in den 1980er Jahren mit einer neuen privatisierten Wohnungsbaupolitik, die Hauseigentümerschaft ermöglichen und fördern soll. Dazu wurden staatliche Subventionen und spezielle Hypotheken für die unteren Einkommensklassen geschaffen, sowie Preislimits und Steuerfreiabkommen für die privaten Baufirmen eingeführt. Besonders seit der Baukrise zu Beginn des Jahrtausends setzen private Konstruktionsfirmen auf den sozialen Wohnungsbau. Seitdem entstehen massenweise großflächige, standardisierte Wohnsiedlungen an den Rändern Bogotás. Bewohner_innen sind Niedrigverdiener_innen, die zuvor in informellen Siedlungen lebten. Für die staatlich subventionierten Hypotheken, mit denen sie sich einkaufen, müssen Käufer_innen ein festes Arbeitsverhältnis nachweisen, was die Hälfte der kolumbianischen Bévölkerung ausschließt. Basierend auf mit diversen Forschungsmethoden in neun verschiedenen Sozialwohnungsbaugebieten erhobenen Daten, analysieren wir in diesem Artikel die gegenwärtige Wohnungsbaupolitik Kolumbiens und ihre Folgen. Wir argumentieren, dass die neue Politik tatsächlich mehr Menschen Zugang zu Eigentumswohnungen verschafft. Gleichzeitig zeigen wir, dass ohne weiterreichende soziale Reformen die Gefahr besteht, dass sich die isoliert liegenden Wohnsiedlungen in urbane Ghettos verwandeln, die ihre Bewohner_innen weiterhin stigmatisieren. Zugang zu Bildung, Jobs, Kultur und anderen urbanen Ressourcen ist notwendig, um langfristige soziale Mobilität zu ermöglichen bzw. zu garantieren.
\end{abstract}

Ersteinreichung: 20. August 2018; Veröffentlichung online: 29. November 2019

An English abstract can be found at the end of the document.

\section{Einleitung}

Der Begriff, sozialer Wohnungsbau' bezeichnet im angelsächsischen Raum sowie in lateinamerikanischen Ländern Wohnraum, der vor den Gesetzen des Marktes geschützt ist (Girola 2007). In der Regel handelt es sich dabei um direkte staatliche Maßnahmen zur Beschaffung von Wohnraum für die ärmsten Bevölkerungsschichten, der nicht die Form von Privateigentum annimmt. Diese Art von öffentlichen Interventionen ist jedoch in den letzten Jahren im Zuge des weltweiten Abbaus des Wohlfahrtsstaates und des Siegeszuges neoliberaler Wirtschaftspolitik immer weiter verdrängt worden.

In Kolumbien fand ein solcher Umbruch in der sozialen Wohnungsbaupolitik schon vor mehr als zwei Jahrzehnten statt. Während der Staat zunächst noch als Vermittler von Wohnraum agierte, kam es sehr bald zu einer gänzlich auf den Markt ausgerichteten Politik (Bermúdez et al. 2012; 
Cuervo/Jaramillo 2009; Parias 2012; Saldarriaga Roa/Carrascal 2006). Ermutigt durch internationale Organisationen wie den Internationalen Währungsfonds (IMF), wurde die Rolle des Staates in der Sozialpolitik reduziert. Ähnlich wie schon in Chile praktiziert, sollte Hauseigentümer_ innenschaft ermöglicht und gefördert werden. Zu diesem Zweck führte Kolumbien staatliche Subventionen für die unteren Einkommensklassen, sowie Preislimits und Steuerfreiabkommen für private Baufirmen ein.

Konstruktionsfirmen erkannten das Gewinnpotential des staatlich subventionierten sozialen Wohnungsbausektors vor allem in der Baukrise Anfang der 200oer Jahre. Seit dieser Zeit schießen an den Rändern der großen kolumbianischen Städte, allen voran in Bogotá, massenweise großflächige, standardisierte Wohnsiedlungen in die Höhe. Deren Bewohner_innen gehören zu den unteren Einkommensschichten und haben zuvor mehrheitlich in informellen Siedlungen gelebt.[1] Staatlich subventionierte Hypotheken helfen ihnen beim Wohnungskauf. Der Zugang zu derartiger Finanzierung ist allerdings mit dem Nachweis eines festen Arbeitsverhältnisses verknüpft, was de facto die Hälfte der kolumbianischen Bevölkerung ausschließt.[2] Dieser Umstand verdeutlicht, dass die heutige Wohnungsbaupolitik des Landes weniger das Ziel hat, den ärmsten Bevölkerungsgruppen Zugang zu Wohnraum zu verschaffen, als vielmehr die Wirtschaft des Landes anzukurbeln. Was sind die Folgen einer solchen wirtschaftlich ausgerichteten Sozialwohnungsbaupolitik? In diesem Artikel diskutieren wir die realen Auswirkungen und Probleme der sozialen Wohnungsbaupolitik in Kolumbien. Wie funktioniert sie? Wer hat Zugang zu dem so geschaffenen Wohnraum und wie lebt es sich in den neuen Siedlungen? Was sind die sozialen, räumlichen und ökonomischen Auswirkungen dieser Politik - für die Menschen und für die Stadt?

Obwohl es sich nicht verneinen lässt, dass die Strategie des privat-finanzierten Wohnungsbaus mehr Menschen Zugang zu eigenem Wohnraum ermöglicht hat, zeigen wir hier die (strukturellen) Probleme der neuen einheitlichen Wohngebiete in den städtischen Randlagen auf: Strikte Nutzungs- und Verhaltensregeln in den Siedlungen sind nicht auf die Bedürfnisse der Bewohner_ innen ausgerichtet. Abgelegen und kaum in urbane Strukturen eingebunden, besteht die Gefahr, dass die neuen Wohngebiete sich zu Ghettos entwickeln, die die extreme sozio-ökonomische und räumliche Stratifizierung der kolumbianischen Gesellschaft noch weiter vertiefen. Wir zeigen, dass die Politik des privatisierten Sozialwohnungsbaus zu kurz greift, da sie nichts an der tiefsitzenden, kulturellen Wirklichkeit der kolumbianischen Klassengesellschaft und den damit verbundenen Vorurteilen ändert. Das Fallbeispiel Kolumbien macht deutlich, dass die Schaffung von Wohnraum wichtig für das Wohlergehen sozial schwacher gesellschaftlicher Schichten ist. Ohne weitere strukturelle Veränderungen, vor allem sichere Arbeitsmöglichkeiten, Zugang zu Bildung und sozialen Dienstleistungen sowie eine Einbindung in den urbanen Raum, ist der langfristige Erfolg solcher Maßnahmen zur Armutsbekämpfung jedoch eher begrenzt beziehungsweise zweifelhaft.

Basierend auf einer sechsmonatigen Studie von Sozialwohnungsbauquartieren in Bogotá, die Umfragen, Interviews und ethnografische Beobachtungen einschloss, diskutieren wir im Folgenden zunächst die sich wandelnde soziale Wohnungspolitik Kolumbiens. Danach erläutern wir die 
Sozialwohnungsbaupolitik im Zusammenhang mit der strukturellen und kulturellen sozialen Ungleichheit sowie die Probleme der sozialen Mobilität im Land. Die Diskussion des Lebens in den Sozialwohnungsbauquartieren, einschließlich ihres Designs und Layouts sowie der Probleme des Zusammenlebens, veranschaulicht, wie problematisch die marktgesteuerte Sozialwohnungsbaupolitik Kolumbiens ist.[3]

\section{Wohnungsbaupolitik in Kolumbien: Von der Armutsbekämpfung zur Privatisierung}

Sozialer Wohnungsbau ist ein politisches Projekt, wie an der Entwicklung der Wohnungs- und Planungspolitik in Kolumbien deutlich wird. Anfang des zwanzigsten Jahrhunderts war das Hauptziel der kolumbianischen Stadtplanung die ,hygienische Urbanisierung'. Dabei handelte es sich vor allem um Wohnrauminterventionen in Arbeiter_innensiedlungen zur Verhinderung der Ausbreitung von Krankheiten (Zeiderman 2016). In den vierziger Jahren kam dasZiel der Modernisierung hinzu. Nicht nur das Garden-City-Modell von Ebenezer Howard, sondern auch die Diskussionen des CIAM (Congrès internationaux d'architecture moderne) über Wohnraum als ,Existenzminimum und die Gewährleistung gesundheitlicher Mindeststandards beeinflussten nun den Wohnungsbau, sowohl was die Form von Arbeiter_innenvierteln anging als auch die Pläne zur Modernisierung von Städten generell (Bermúdez et al. 2012).

1939 schuf die kolumbianische Regierung das Territoriale Kreditinstitut ICT (Instituto de Credito Territorial), das während des größten Teils des 20. Jahrhunderts Kolumbiens führende staatliche Institution zur Konstruktion von Wohnraum für die ärmsten Bevölkerungsschichten war. Der so geschaffene Wohnraum war allerdings nicht ausreichend, besonders ab den sechziger Jahren, als die Land-Stadt-Migration und der Wohnraumbedarf dramatisch anstiegen. Die Folge war das rapide Anwachsen informeller Siedlungen an den Rändern großer kolumbianischer Städte (Parias 2012). Mit finanzieller Unterstützung internationaler Organisationen, und ähnlich wie andere Regierungen in der Region, ging die kolumbianische Regierung daher dazu über, solche Siedlungen zu regulieren. Gleichzeitig aber grenzte die weiter vorherrschende modernistische Stadtplanung einen Großteil der kolumbianischen Bevölkerung aus, indem sie „die Instrumente zur Marginalisierung und Kriminalisierung der armen Bevölkerung unter dem Vorwand der Verbesserung des städtischen Lebens" bereitstellte (Pérez 2010: 58, Übers. d. A.).

Von 1942, dem Jahr, in dem das ICT Wohnungen sowohl in der Stadt als auch auf dem Land zu bauen begann, bis in die siebziger Jahre nahm der soziale Wohnungsbau sowohl die Form großer staatlicher Bauprojekte als auch der Förderung von Selbstbauprojekten an.[4] Zu dieser Zeit gab es allerdings schon andere Neuerungen: Zum einen wurden nun Hochhäuser gebaut und zum anderen begannen private Firmen damit, sozialen Wohnraum zu konstruieren. Gleichzeitig hatte sich das Ziel der staatlichen Politik verschoben. Beeinflusst durch die Politik multilateraler Organisationen in Lateinamerika verdrängte die Armutsbekämpfung die bisherigen Modernisierungsideale. Erst in diesem Zusammenhang entstand die explizite Kategorie des ,sozialen 
Wohnungsbaus' und ,der_die Arbeiter_in'wurde durch ,die Armen“als Objekt staatlicher Interventionen ersetzt (Zeiderman 2016).

Weitere Reformen in den siebziger Jahren halfen bei der Konsolidierung der neuen Wohnungsbaupolitik. So führte die Nationalregierung in Zusammenarbeit mit dem Stadtplanungsbüro von Bogotá Baumindeststandards ein, um Familien mit beschränkten wirtschaftlichen Ressourcen „realistische Alternativen zu informellen Urbanisierungen, Landinvasionen und Ähnlichem zu bieten“ (Parias 2012: 75, Übers. d. A.). Dank einer Reihe von Finanzreformen konnten Konstruktionsfirmen, die bislang Privathäuser für den mittleren und höheren Einkommenssektor gebaut hatten, nun zu kapitalwirtschaftlichen Akteuren werden. Zu den Reformen des Finanzsektors gehörte die Schaffung des Systems der UPAC (unidad de poder adquisitivo constante, Konstante Kaufkrafteinheit)[5] als Anreiz zum Sparen für Wohnungsbaukredite und die Entstehung der Spar- und Wohnungsbaugesellschaften (corporaciones de ahorro y vivienda, CAV) als Spezialbanken im Bausektor und für Bausparer_innen (Cuervo/Jaramillo 2009). Der Boom der privatwirtschaftlichen Konstruktion von Sozialwohnraum in der Form standardisierter Großprojekte begann allerdings auch dank einer wachsenden Mittelschicht, die bereit war, diese Art von Wohnraum zu beziehen und in den preisgünstigeren neuen peripheren Lagen zu leben.

Das Anwachsen städtischer Kriminalität im Zusammenhang mit dem Drogenhandel und die Angst um Sicherheit führten in den achtziger Jahren zur Entstehung und Konsolidierung der bis heute in den meisten kolumbianischen Städten überwiegenden Form des Wohnens: geschlossene Wohnviertel (conjuntos cerrados, Gated Communities) für alle Einkommensschichten (mit Ausnahme der illegalen und informellen Siedlungen). Auf internationaler Ebene wurde zu dieser Zeit bereits die Notwendigkeit diskutiert, das Recht auf Wohnraum in Form des einfachen ,Dachs über dem Kopf durch die Schaffung von Wohnraum inklusive Infrastruktur, Dienstleistungen, Ausstattung und lebenswerter Umgebung zu ersetzen (UN Habitat 1976). In Bogotá entstanden in dieser Zeit zwei Großprojekte, die in der Tat Infrastruktur, Dienstleistungen, Parks und Einrichtungen anboten: Ciudad Salitre für den Mittelstand und Ciudadela Colsubsidio für den sozialen Wohnungsmarkt. Da das Angebot an Sozialwohnungen jedoch weiterhin unzureichend war, weitete sich in dieser Zeit der informelle Markt aus; die zentralsten und ältesten Stadtteile informellen Ursprungs verdichteten sich und der Mietmarkt wuchs an. Gleichzeitig konsolidierten sich illegale Wohnraumanbieter_innen an den Peripherien (Parias 2012).

Im Zuge der wirtschaftlichen Liberalisierung und Dezentralisierung der neunziger Jahre kam es zu weiteren Reformen, die den sozialen Wohnungsbau bis heute beeinflussen. Zum einen wurde die, soziale und ökologische Funktion' von Eigentum in das Gesetz zur urbanen Reform aufgenommen. Dies ermöglichte den Kauf großer Landflächen für den Bau sozialen Wohnraums. Zum anderen beinhaltet die Verfassung von 1991 das Recht auf menschenwürdiges Wohnen. Gleichzeitig ist die Rolle des Staates als Institution festgelegt, die dieses Recht durch die Förderung von sozialen Wohnungsbauplänen, nicht aber durch direkten Wohnungsbau umsetzt.

Die Vorgaben der Verfassung formten die Grundlage für das Gesetz Nr. 3 von 1991, bekannt als VIS-Gesetz (vivienda de interes social, 
Sozialbauwohnungen). Dieses Gesetz schaffte die bislang existierenden Institutionen und Programme des sozialen Wohnungsbaus ab und ersetzte sie durch Direktsubventionen. Das heißt, seit dieser Zeit bietet der Staat individuellen Haushalten die Teilfinanzierung des Wohnungskaufs an.[6] Gleichzeitig löste die Nationalregierung die ICT auf und schuf stattdessen das INURBE (Instituto Nacional de Vivienda de Interés Social y Reforma Urbana, Nationales Institut für Sozialwohnungen und Urbane Reform), um die Subventionen für den sozialen Wohnungsbau zu verwalten. Außerdem endete das auf den Konstruktionssektor spezialisierte Finanzierungssystem und der Rest des Finanzsektors wurde in der Multibanca vereint (Cuervo/ Jaramillo 2009). Auf diese Weise wurde der Großteil des sozialen Wohnungsbaus in den Privatsektor verlagert, was dem Bausektor entgegenkam, da Anfang der neunziger Jahre die Wohnraumnachfrage höherer Einkommensgruppen gesättigt, und der Immobilienmarkt in einer Krise war. Die Möglichkeit, in großen Mengen preiswerte Wohnungen für die unteren Einkommensklassen zu bauen, erlaubte es den Firmen, die Krise zu überstehen (Parias 2012).[7]

Die Verknüpfung der öffentlichen Hand mit dem Privatsektor wurde noch deutlicher während der ersten Amtszeit von Bogotás Bürgermeister Enrique Peñalosa (1998-2000), der mit der Bauträgergesellschaft Metrovivienda ein ,industrielles und kommerzielles Unternehmen der Stadt“[8] gründete. Metrovivienda kauft Land, erschließt es und verkauft es dann weiter an private Bauunternehmen, um Sozialwohnungen zu bauen. Das Ergebnis sind bislang zwei große Wohngebiete am südwestlichen Stadtrand, El Recreo und Porvenir. Obwohl Metrovivienda auch öffentliche Träger_innen zulässt (Cuervo 2013), wird der Hauptanteil der Sozialwohnungen von privaten Unternehmen gebaut. Diese liegen fast ausschließlich an den Stadträndern und in umliegenden Gemeinden.

Präsident Alvaro Uribe führte diese Strategie fort. In seiner zweiten Amtsperiode (2006-2010) schuf die Nationalregierung das Format der „Sozialen Makroprojekte nationalen Interesses“ (macroproyectos de interés social nacional)[9] mit dem Ziel, die Verfügbarkeit von zu entwickelndem Land zu erleichtern und das Wirtschaftswachstum durch Förderung des Bausektors zu beschleunigen. Diese Politik ging maßgeblich auf die Lobbyarbeit des Bausektors zurück. Nachdem die Strategie 2010 vom Verfassungsgericht als unzulässig erklärt wurde, rief die Nationalregierung 2011 die Politik der „Makroprojekte der zweiten Generation“ (macroproyectos de segunda generación)[10] aus. Seitdem besteht die Wohnungspolitik der nationalen Regierung aus drei Programmen, die Begünstigte in verschiedene Gruppen unterscheidet: Für Menschen in, verletzlicher Situation ' (condiciones de vulnerabilidad) gibt es das ,Kostenlose Wohnungsprogramm'. Das sind Menschen, die ihre Wohnstätte durch den Bürgerkrieg oder Naturkatastrophen verloren haben. Haushalte mit ,Sparkapazität‘ und einem Einkommen von weniger als zwei gesetzlichen Mindestlöhnen (26,8 Prozent aller Haushalte Kolumbiens) sind die Zielgruppe des „Wohnungsprogramms vorrangigen Interesses für Sparer" (Vivienda para Ahorradores, VIPA). Und für die ,Mittelklasse', definiert als Haushalte mit einem Einkommen zwischen zwei und vier gesetzlichen Mindestlöhnen (51,4 Prozent der Bevölkerung), gibt es das Programm „Mein Haus Jetzt“ (Mi Casa Ya) (Minvivienda 2015). Sozialer 
Wohnungsbau in Kolumbien schließt demnach explizit die ,Mittelklasse', das heißt Haushalte mit Einkünften von 1.562.00o COP (ca. 440 Euro, Stand 2019), mit ein.

Der Nationale Entwicklungsplan der ersten Regierung von Juan Manuel Santos (2010-2014) fasst die aktuelle Denkweise zum Bau von Wohnraum in Kolumbien zusammen:

„Konstruktion ist unsere Antwort, um den Anstieg der Wohnraumnachfrage, der durch den wirtschaftlichen Aufschwung erzeugt wird, zu nutzen, um Arbeitsplätze besonders in den städtischen Gebieten des Landes zu schaffen. Auf diese Weise kann der Wohnungssektor angesichts seiner vielfältigen Verbindungen zu verschiedenen anderen wirtschaftlichen Aktivitäten die Wirtschaft ankurbeln und gleichzeitig Wachstum und Beschäftigung fördern. Dies, ohne die sozialen Auswirkungen zu vergessen, die kolumbianische Haushalte durch mehr und bessere Lebensbedingungen erfahren." (DNP 2011: 52; Übers. d. A.)[11]

In diesem Zitat wird die wirtschaftliche Ausrichtung der Sozialwohnungsbaupolitik deutlich: Die Betonung liegt weniger auf der Schaffung von Wohnraum als auf der Schaffung von Arbeitsplätzen und der Förderung des Bausektors, was die Unterordnung des sozialen Aspektes der Wohnungspolitik unterstreicht. In der Tat wird die Wohnungsbaupolitik Kolumbiens seit Anfang der neunziger Jahre von der Wirtschaftskommission für Lateinamerika und die Karibik (CEPAL) als „marktorientierte Politik“ beschrieben (Anzellini et al. 2012; Bermúdez et al. 2012; Cuervo/Jaramillo 2009; Parias 2012; Saldarriaga Roa/Carrascal 2006) und folgt dem globalen Trend der ,Finanzialisierung von Wohnraum'.[12] Diese Finanzialisierung beinhaltet den Rückzug des Staates aus dem Wohnungssektor, den allgemeinen Konsens, dass Wohneigentum ein Synonym von Freiheit und sozialem Aufstieg ist, und die ,Sozialisierung von Kredit', das heißt, dass neue Konsument_innen mit niedrigem und mittlerem Einkommen Zugang zu Finanzkreisläufen bekommen (Aalbers 2017; Rolnik 2013). Diese Sichtweise auf Wohnraum als in globale Finanzmärkte eingebundenes Anlagevermögen ist charakteristisch für das ökonomische Denken der letzten Dekaden (Rolnik/Rabinovich 2014). Es handelt sich also nicht um eine für Kolumbien- oder Lateinamerika spezifische Strategie, sondern, wie Raquel Rolnik und Lidia Rabinovich (ebd.) zeigen, um eine globale Tendenz. Es ist jedoch gerade die Finanzialisierung von Wohnraum, die weltweit Wohnungseigentümer_innenschaft - auch für die Mittelklasse - in Frage gestellt beziehungsweise in eine Krise gestürzt hat (siehe z. B. Forrest/Hirayama 2011). Demnach stellt sich die Frage, wie erfolgreich oder nachhaltig diese Politik sein kann.

\section{Schichtzugehörigkeit, Sozialwohnungsbau und soziale Mobilität}

Vorangehend haben wir gesehen, wie der privatisierte soziale Wohnungsbau in Kolumbien vor allem seit den neunziger Jahren vornehmlich als Wirtschaftsmotor und nicht als sozialstaatliche Verantwortung konzipiert worden ist. Dies geht einher mit dem Regierungsprojekt, Kolumbien in ein 
Mittelklasseland zu verwandeln. Wie Präsident Juan Manuel Santos 2015 als Bilanz seiner ersten Amtszeit deklarierte: „Was wir seit 2010 wollten, war ein Land mit weniger Armut, mehr Arbeitsplätzen, und mehr Sicherheit. Und heute können wir mit großem Stolz sagen, dass wir schon ein Land der Mittelklasse sind.“ (Portafolio 2015, Übers. d. A.) Santos bezog sich damit auf eine Studie der Interamerikanischen Entwicklungsbank (IADB), nach der 55 Prozent der kolumbianischen Bevölkerung zur Mittelklasse gehören. 2002 verdiente die Hälfte aller Kolumbianer_innen weniger als vier Dollar am Tag, was von der Weltbank als Armut definiert wird (World Bank 2018). Davon lebten 17,7 Prozent in extremer Armut. 2015 lag der Armutsanteil bei 28,5 Prozent und der der extremen Armut bei 8,1 Prozent (Semana 2015).

Wie nachhaltig ist aber die soziale Mobilität der letzten Dekade? Der Interamerikanischen Entwicklungsbank zufolge drohen 55 Prozent der kolumbianischen Mittelschicht wieder zurück in die Armut zu fallen (Semana 2015). Darüber hinaus wird vor allem auch diskutiert, was ,Mittelklasse“ im lokalen Kontext genau bedeutet. Rafael de la Cruz, Vertreter der IADB in Kolumbien, meint dazu: „Die Mittelklasse hier ist keine mit Garten und Swimming Pool. Wir sehen sie eher als eine Gruppe, die die Armut überwunden hat und nun Teil ihres Einkommens zum Erwerb von Konsumgütern benutzen kann.“ (ebd.). Dies sei näher an der nationalen Realität. Die Mittelklasse in Kolumbien kaufe sich ein Auto und ein Haus, habe eine Kreditkarte und ein Smartphone, und könne an die Bildung der Kinder und die Sicherheit der Familie denken (ebd.). Offensichtlich liegt hier ein Verständnis von Mittelklasse zu Grunde, das an konsumorientiertem Verhalten, und allem voran, dem Hauskauf festgemacht ist.

Die Diskussion ähnelt der von Charles Klein et al. (2018) erörterten Situation in Brasilien, wo Armutsbekämpfungsmaßnahmen in den letzten Dekaden angeblich 35 Prozent der armen Bevölkerung in die Mittelklasse hat aufsteigen lassen. Dieser Analyse liegt, wie in Kolumbien, eine auf Einkommen und Konsumverhalten beruhende Definition der Mittelklasse zugrunde, was von brasilianischen Sozialwissenschaftler_innen als zu kurz greifend kritisiert wird, zumal eindeutige Unterschiede und Diskriminierungen zwischen der ,neuen ' und der ,alten' Mittelklasse fortbestehen. Die Autoren schlagen daher vor, die neue Mittelklasse besser als ,vormalig Arme zu bezeichnen (ebd.).

Ebenso ist es höchst fraglich, ob in Kolumbien die Hauseigentümer_innenschaft allein tiefer liegende, strukturelle Ungleichheiten und Vorurteile beheben und somit eine nachhaltige soziale Mobilität der, vormalig Armen“ fördern kann. Umso mehr als Bogotá, ähnlich wie andere kolumbianische und südamerikanische Städte, sehr stark stratifiziert ist, allerdings nicht nur sozio-ökonomisch sondern auch räumlich. Dies geht auf ein spezielles administratives System der öffentlichen Leistungen zurück: Das gesamte Stadtgebiet ist in Schichten (estratos) von 1 (niedrig) bis 6 (hoch) eingeteilt, die auf einer offiziellen Schätzung des sozioökonomischen Wertes eines Stadtgebietes beruhen.[13] Diese Schichten sind die Grundlage für die Berechnung der Wasser-, Abwasser- und Stromkosten, wobei höhere Einkommensschichten proportional höhere Preise zahlen und dadurch die untersten Einkommensschichten subventionieren.[14] Zudem dient die Schichtzuordnung als Grundlage für die Berechnung der Grundsteuern. Abgesehen davon, dass dieses System die sozioökonomischen Lebensumstände der Bogotáner_innen übermäßig 
vereinfacht, beeinflusst die Klassifizierung den Immobilienmarkt, wenn Personen zum Beispiel bewusst in einem niedriger bewerteten Gebiet Wohnraum erwerben und darauf spekulieren, dass der Wert dieses Gebiets mit der Zeit steigt. Darüber hinaus trägt das System zur Assoziierung von Stadtgebieten mit bestimmten sozioökonomischen Schichten bei. So werden zum Beispiel Afro-Kolumbianer_innen im wohlhabenden Norden Bogotás als „Fremdkörper` wahrgenommen (Castro 2013), und Haushaltsangestellte, die dort nach Arbeitsende ohne ihre Arbeitsuniform in den Supermarkt gehen, berichten, von Ladendetektiv_innen verfolgt zu werden (Fleischer/ Marin 2019).

In der Tat ist das System der estratos Teil der städtischen Alltagskultur geworden und wird nicht nur mit der Art und Qualität von Dienstleistungen und Angeboten in den Stadtteilen assoziiert, sondern dient auch der Charakterisierung von Personen: Jemand ist ,estrato uno' (Schicht 1), wenn ihm oder ihr bestimmte,Qualitäten` zu fehlen scheinen. Dabei kann es sich um Geschmack, Manieren oder Wissen handeln; das Kriterium sind Werte, die mit der wachsenden städtischen Mittel- und Oberschicht verbunden sind oder von diesen als wünschenswert angesehen werden.

Es ist besonders diese Assoziierung von Lokalität und Schichtzugehörigkeit, die das Anwachsen der Sozialwohnungsquartiere in den Randgebieten der Stadt, die mit den niedrigen Schichten assoziiert sind, so problematisch macht. Der fortdauernde Klassismus, die tiefsitzende Diskriminierung von bestimmten Wohngebieten und ihren Bewohner_innen, wird weder durch die privatisierte Wohnungsbaupolitik noch die strikten Verhaltensregeln in den neuen Wohngebieten adressiert - geschweige denn verändert. Dies wurde deutlich in dem Kommentar einer leitenden Angestellten einer der größten kolumbianischen Sozialwohnungsbaufirmen, die sich auf die neu gebauten Wohnviertel in den Randlagen der Hauptstadt als „Land der Comanchen“ (el territorio de los comanches) bezog.[15]

\section{4. „El Territorio de los Comanches“: Struktur und Lage der Sozialwohnungsbauviertel}

Sozialwohnungsbauviertel sind fast ausschließlich an den (südlichen) Stadträndern angesiedelt, wo preiswerteres Land den privaten Konstruktionsfirmen höhere Gewinnspannen erlaubt (Abb. 1). Sie sind umgeben von anderen, ähnlichen Wohngebieten, Industriegebieten, oder informellen Siedlungen. Oft fehlt es in diesen Randgebieten an Infrastruktur und Transportmöglichkeiten.

Der soziale Wohnungsbau unterscheidet sich von regulären (nicht-subventionierten) Wohneinheiten durch Preislimits: Wohneinheiten dürfen nicht mehr als 75 Prozent des legalen monatlichen Mindestlohns kosten und werden mit von der Regierung subventionierten Krediten verkauft. Um diesen Beschränkungen zu entsprechen, sind die Wohneinheiten extrem klein - zwischen 43 und 73 Quadratmeter. Außerdem werden sie als technikfertige Ausbaustufe (obra gris) verkauft, das heißt ohne jegliche Ausstattung wie Bodenbeläge, Verputz, Kücheneinrichtung oder sanitäre Anlagen. Häuser haben zudem oftmals nur ein oder zwei Stockwerke, sind aber so konstruiert, dass Bewohner_innen selbst aufstocken können. 
Abb. 1 Sozialwohnungsbausiedlungen am Südrand von Bogotá (Foto: Autorinnen)
Abb. 2 und 3 Standardisierte Sozialwohnungsbauviertel (Fotos: Autorinnen)

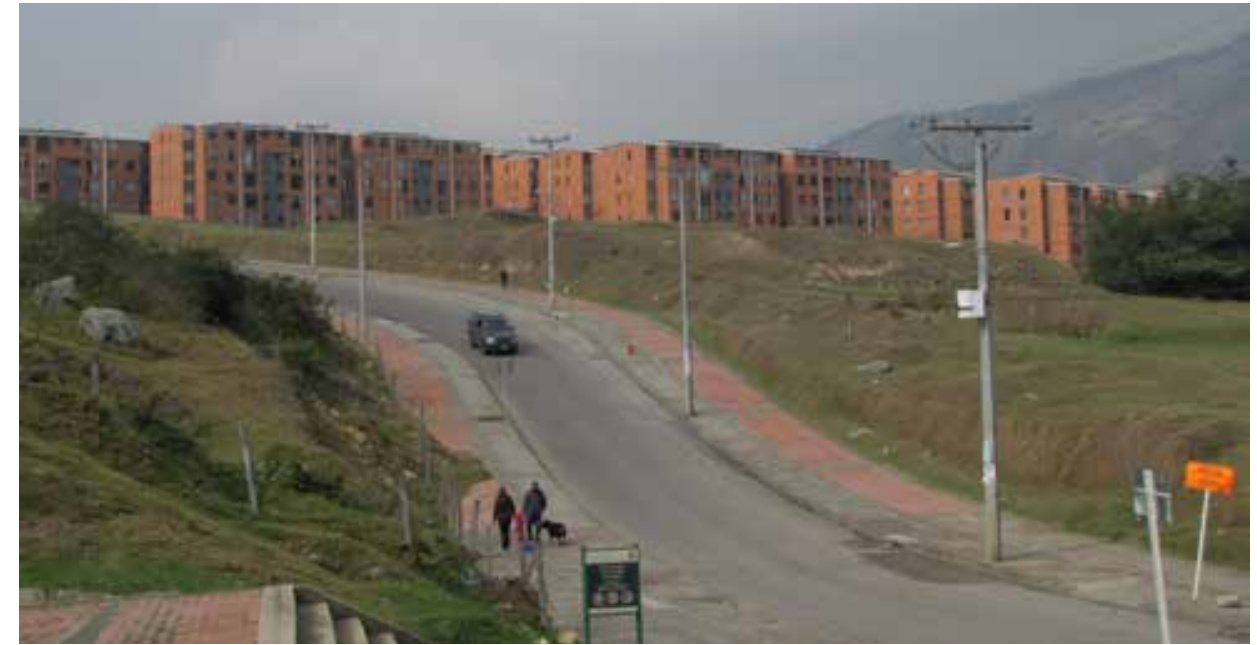

Um ihre Gewinnspanne weiter zu vergrößern, sparen die Baufirmen wo immer möglich, zum Beispiel in Bezug auf das Design der Wohngebiete selbst. Das Ergebnis sind extrem standardisierte Viertel. Form, Layout und Innenarchitektur sind fast identisch: Von hohen Mauern oder Zäunen umschlossene, von Wachpersonal gesicherte rote Backsteinsiedlungen, mit kaum Rasenflächen zwischen den sechs- bis acht-stöckigen gleichförmigen Gebäuden (Abb. 2 und 3). Bewohner_innen haben - wenn überhaupt - nur sehr eingeschränkte räumliche Möglichkeiten, um miteinander zu interagieren oder Kontakte zu knüpfen. Extrem dünne Wände, das Fehlen von Dämmmaterial sowie das generelle Layout der Apartmentgebäude bieten keinerlei Schutz gegen Lärmbelästigung, was durch die hohe Wohndichte pro Quadratmeter noch verschlimmert wird.[16] Zudem sind die Gebäude extrem dicht aneinander gebaut und parallel ausgerichtet: Der Eingangsbereich eines Gebäudes blickt auf die Rückseite des nächsten, womit die tägliche soziale Interaktion der Bewohner_innen verhindert wird. Trotz dieser Einschränkungen bieten die Wohnprojekte Zugang zu Privateigentum und sind daher attraktiv für die unteren Einkommensklassen mit unterschiedlichen sozialen, kulturellen und ethnischen Hintergründen. Laut unserer Umfrage lebten etwa siebzig Prozent der Bewohner_innen vorher nicht in Wohnungen, sondern in selbstgebauten Häusern in informellen Siedlungen.

Um eine Wohnung in den sozialen Wohnungsbauquartieren zu kaufen, bedarf es einer Anzahlung aus eigenen Mitteln, die unterstützt wird
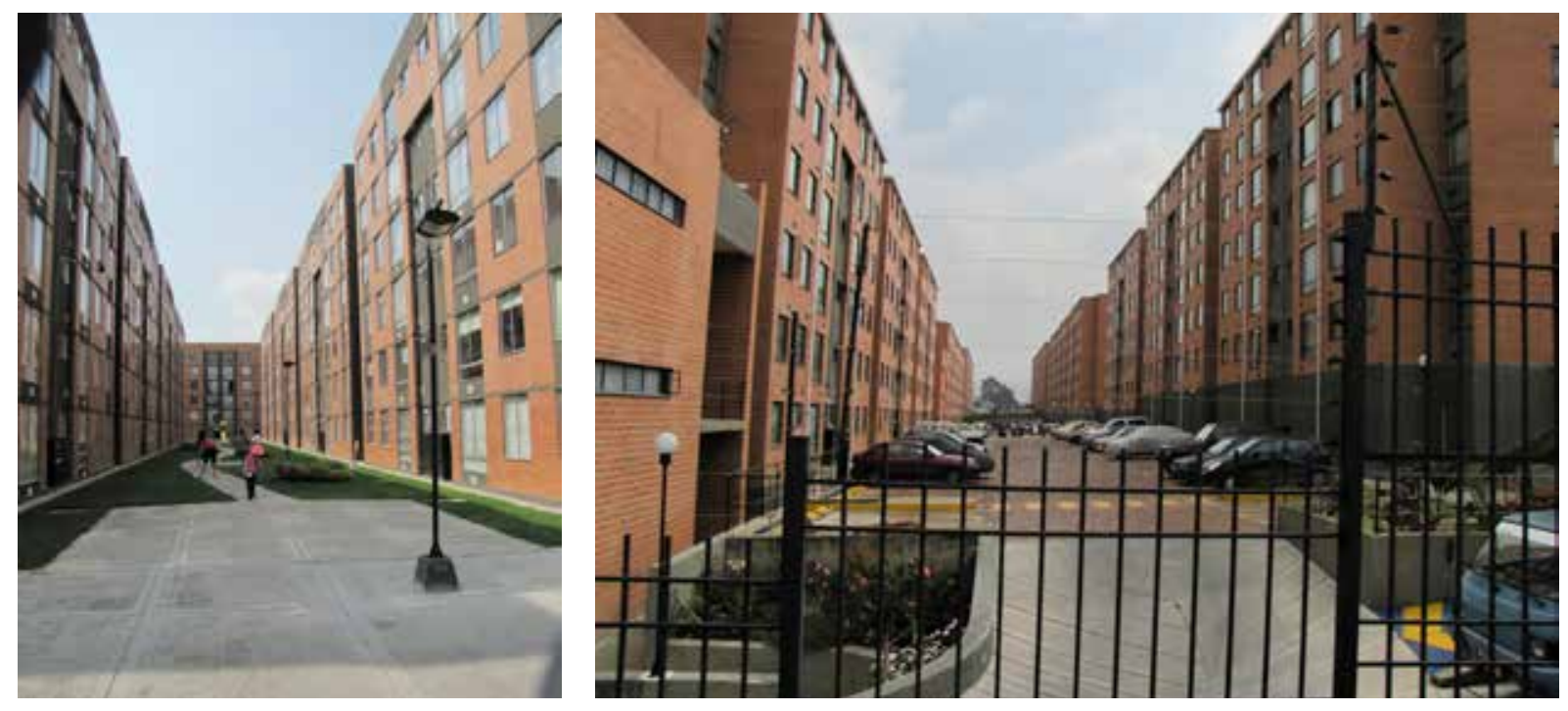
von Regierungssubventionen. Danach zahlen Bewohner_innen den Rest des Kaufpreises in monatlichen Ratenzahlungen. Trotz der Preislimits auf Sozialbauwohnungen beantragt die große Mehrheit der Käufer_innen einen Finanzierungskredit. Um an einen solchen Kredit zu kommen, müssen Bewerber_innen allerdings ein geregeltes monatliches Einkommen und Ersparnisse nachweisen. Dies macht es für Selbständige extrem schwer, an einen Kredit zu kommen. Noch schwieriger ist es für Menschen im informellen Sektor, was 2016 fast die Hälfte der kolumbianischen Bevölkerung ausmachte (DANE 2018). Aber sogar formell Angestellte haben mitunter Schwierigkeiten mit ihren Kreditratenzahlungen. Kolumbiens Arbeitsmarktpolitik erlaubt es Firmen, ihre Angestellten ohne große Hindernisse von heute auf morgen zu entlassen; dies betrifft besonders den niedrig qualifizierten, niedrig bezahlten Einkommenssektor. Zudem haben Niedrigverdiener_innen weniger Ressourcen (Besitz, Ersparnisse oder finanziell besser gestellte Verwandtschaft) auf die sie in Krisenzeiten zurückgreifen könnten.

All dies bedeutet, dass die Privatisierung von Kolumbiens sozialem Wohnungsmarkt einen Großteil der Bevölkerung, und vor allem Niedrigverdiener_innen mit dem größten Bedarf an Wohnraum von vornherein ausschließt, oder aber es extrem schwierig für sie macht, sozialen Wohnraum zu erwerben. Diejenigen, die es schaffen, sich in die neuen Wohnanlagen einzukaufen, sind durch das marktwirtschaftlich bedingte ,kosteneffektive Layout und die abgelegene Lage der Wohnkomplexe von der Stadt selbst separiert. Mit den Kreditratenzahlungsverpflichtungen erweist sich das ,soziale‘ Element im sozialen Wohnungsbau Kolumbiens als minimal. In gewisser Hinsicht wird also durch die Ausrichtung der kolumbianischen Sozialbaupolitik selbst die Marginalisierung oder Ausgrenzung der niedrigen Einkommensschichten verschärft.

\section{Probleme und Regulierung des Zusammenlebens in Sozialbauquartieren}

Die Lage und das Design der sozialen Wohnquartiere, zusammen mit der oft recht prekären ökonomischen Situation der Bewohner_innen, die zudem vielfach noch nie in einer vergleichbaren Wohnsituation gelebt haben, trägt dazu bei, dass es verschiedene Probleme und Spannungen in den neuen Wohnsiedlungen gibt, nicht nur zwischen den Bewohner_innen, sondern auch mit den Verwalter_innen, den Baufirmen und den lokalen Behörden.

Im Alltag des Siedlungslebens beklagen sich die Bewohner_innen vor allem über Lärm und die Handhabung von Haustieren. Sie streiten sich aber auch mit den Konstruktionsfirmen über strukturelle Probleme der Wohnanlage oder unerfüllte Versprechungen. Auf Grund der billigen Bauweise leiden die sozialen Wohnungsbauviertel oft an rapidem Verfall, sowohl der Gebäude als auch des Umfeldes. Hinzu kommen die Nutzung des gemeinschaftlichen Raums beispielsweise durch Straßenverkäufer_innen sowie die Zweckentfremdung von Wohnraum, Kleinkriminalität, Drogenhandel und -konsum, Androhung und Ausübung von Gewalt, und andere Störungen der öffentlichen Ordnung (Abb. 4).

Obwohl uns Bewohner_innen sagten, dass die meisten dieser Konflikte durch die Verwalter_innen der Wohnkomplexe gelöst werden konnten, gab 
Abb. 4 Straßenverkauf (Foto: Autorinnen)

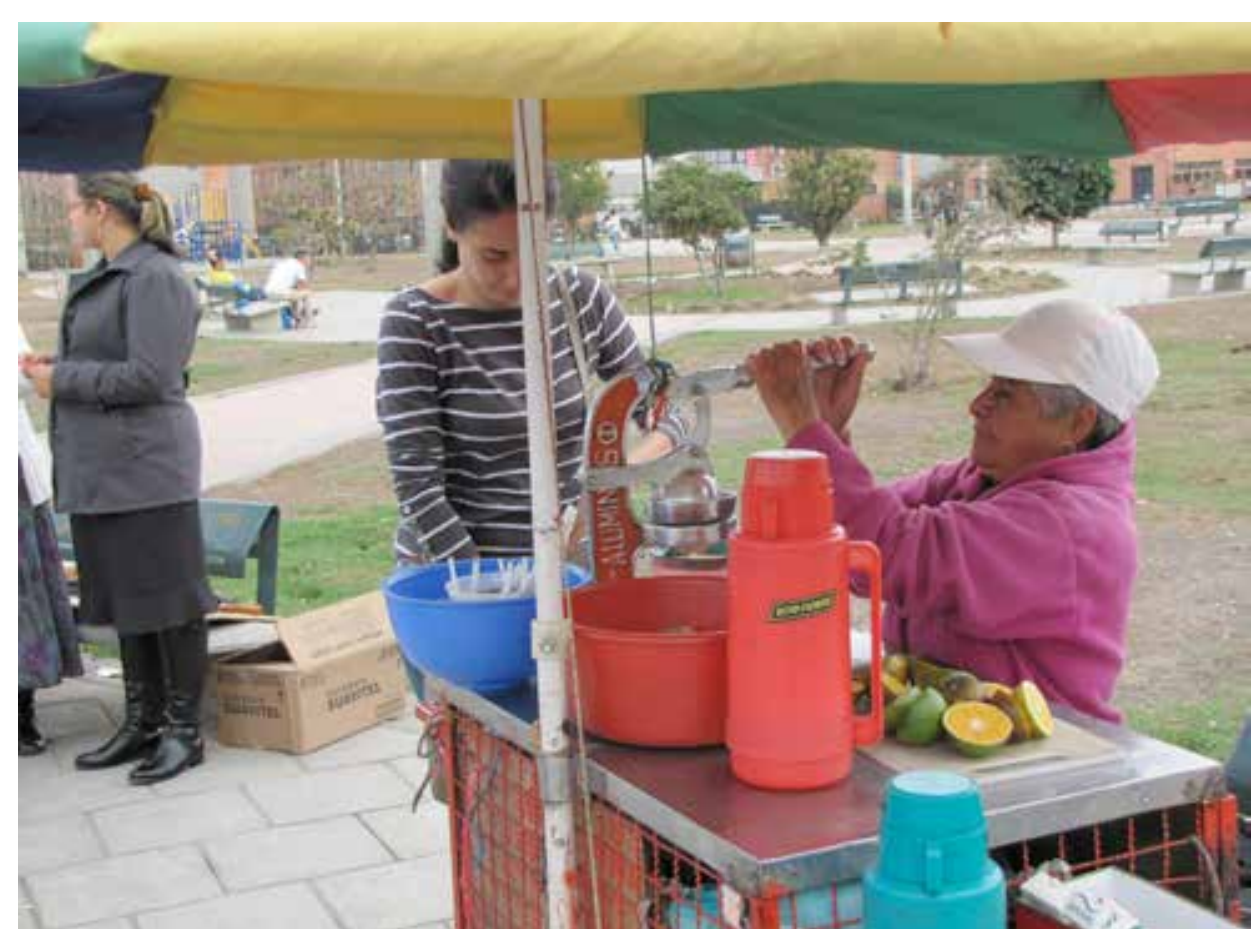

es durchaus auch problematischere Konfrontationen zwischen Nachbar_innen. Während unserer Forschung waren wir zum Beispiel Zeug_innen, wie die Polizei intervenieren musste, weil ein Bewohner einen anderen mit einem Messer bedrohte und ein Verwalter von einem Teenager angegriffen wurde, nachdem dieser ihm verboten hatte, im Hausflur Marihuana zu rauchen. Zudem prahlte ein Bewohner damit, einen anderen mit seiner Pistole bedroht zu haben. In einigen der von uns untersuchten Wohnanlagen war es normal, die Polizei zu rufen, um Konflikte zwischen Bewohner_innen zu lösen. Ein Bewohner kommentierte dies so:

„Meistens kommt die Polizei, verstehst du? Was auch immer das Problem, die Polizei, die Polizei. Ich sage schon, die sollten eine lokale Polizeistation [CAI] hier in unserem Park installieren. (lacht) Die Leute gewöhnen sich daran. Du gehst vorbei und sagst zum Nachbarn dies oder das, und der antwortet: ,Ich klag dich an!' und dann kommt die Polizei. Das sind [gar] keine großen Sachen [die passieren]." (Interview, Bogotá im März 2016, Übers. d. A.)[17]

Um solche und ähnliche Probleme des Zusammenlebens zu beseitigen, sind die Wohnsiedlungen extrem reguliert. Die Verbote und Sanktionen sind in der gesamten Siedlung öffentlich ausgehängt, Videokameras und Wachpersonal kontrollieren nicht nur Besucher_innen, sondern auch Bewohner_innen (Abb. 5-7).[18] Vorschriften gibt es unter anderem für das Recycling von Müll, das Parken von Vehikeln, den Umgang mit Haustieren, die erlaubte Zeit und Dezibelzahl für Musik und anderen Lärm sowie ästhetische Angelegenheiten, wie zum Beispiel dafür, dass keine Wäsche aus den Fenstern gehängt oder kein persönliches Eigentum (Dreiräder, Fahrräder, Möbel, Töpfe) in den gemeinschaftlichen Räumen abgestellt werden darf. Weiterhin wird vorgeschrieben, bis zu welcher Zeit sich Bewohner_innen in den öffentlichen Räumen (innen und außen) aufhalten dürfen, und in einigen Wohnsiedlungen gab es Ausgangssperren für Minderjährige nach 21:0o Uhr. Verstöße gegen diese Regeln werden von der Verwaltung mit 

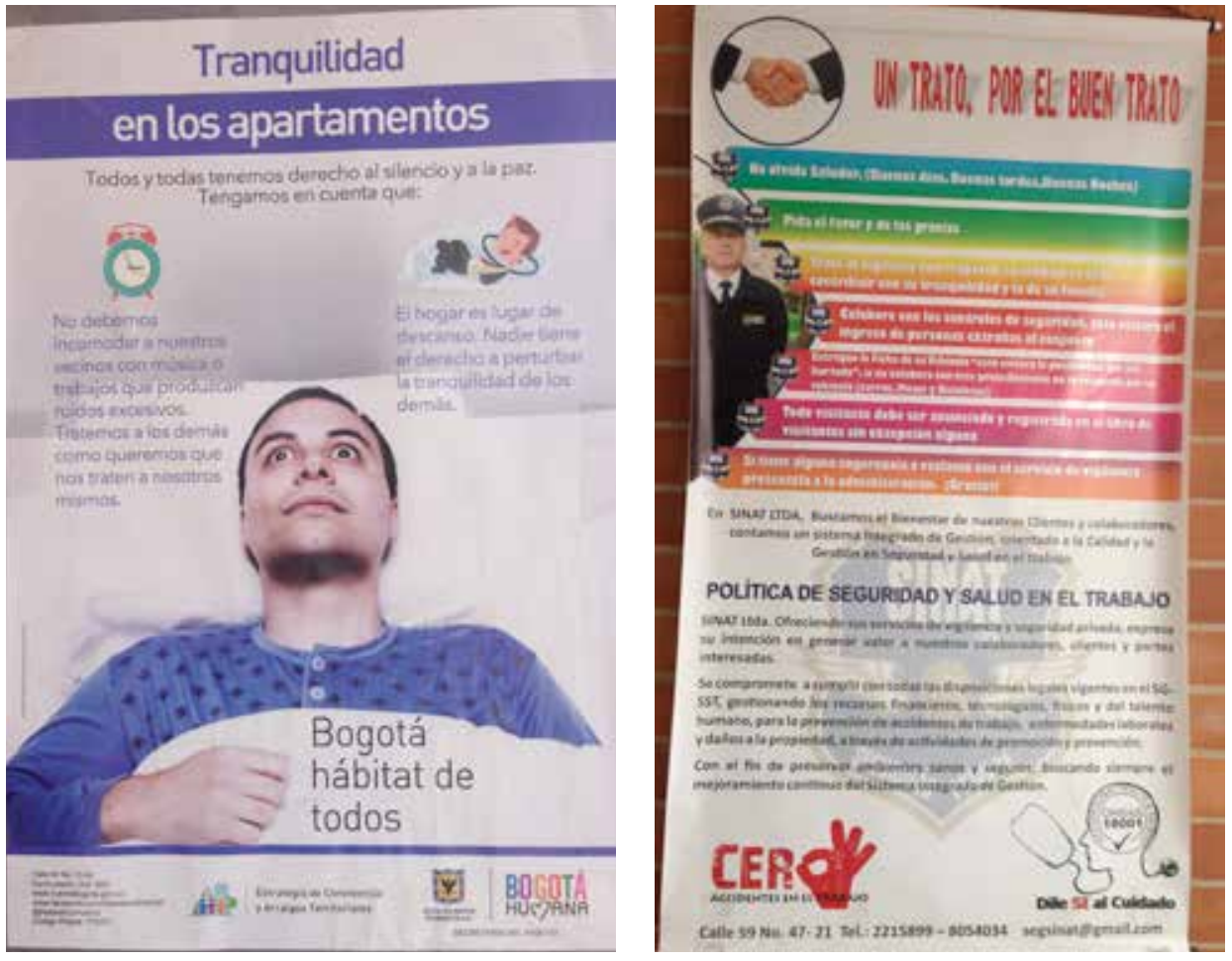

Abb. 5 und 6 Regulierungen (Fotos: Autorinnen)

mündlichen und schriftlichen Abmahnungen, Sanktionen, und Geldbußen geahndet, oder aber es kommt zu ,moralischen' Sanktionen wie der öffentlichen ,Anklage‘ von Verstößen durch Bewohner_innen während der Eigentümer_innenversammlung. Der Verstoß gegen eine der Hauptpflichten der Eigentümer_innen, das Zahlen der Verwaltungsgebühr, wird vielfach (entgegen des Rechts auf Privatheit) in öffentlichen Listen im allgemeinen Eingangsbereich der Siedlung bekanntgemacht; in einer Siedlung wurden orangefarbene Schilder an die öffentlich einsehbaren Postfächer gehängt, auf denen ,Säumig' (moroso) stand (Abb. 8).

Hinter den Vorschriften und strikten Regulierungen des Zusammenlebens steht ein klarer pädagogischer Auftrag: Vor allem den Menschen, die zuvor in informellen Siedlungen gelebt haben, beizubringen wie ,man' in Wohnsiedlungen lebt. Ein Verwalter drückte dies sehr deutlich aus:

„[Das Zusammenleben] leidet, wenn neue Bewohner einziehen, die vorher [...] nicht in horizontalem Eigentum [Wohnsiedlungen] gelebt haben [...]. Diese [Menschen], die aus einer informellen Siedlung kommen, sie versuchen es [...], [aber] bis sie die das Gesetz zum [Leben im] horizontalen Eigentum kennen und sich an die Regeln gewöhnt haben, das kostet immer viel Arbeit. [...] Einige Bewohner kommen aus anderen Wohnsiedlungen, aber andere kommen aus den [informellen] Siedlungen [...] und sind es gewohnt, spät abends Partys zu feiern oder zu jedweder Zeit an die Wand zu klopfen [...]. Aber hier sind das geteilte Wände, Verbindungswände, folglich merkt man alles, es belästigt die Nachbarn. Und was die Musik anbelangt, obwohl es ihnen beim Einzug erklärt wird, einige Eigentümer sind nicht sehr darauf bedacht, den Regeln zu folgen.“ (Interview, Bogotá im März 2016, Übers. d. A.)

Aber auch die Bewohner_innen selbst sind der Überzeugung, dass das Leben in den Wohnvierteln im Vergleich zum Leben in informellen Siedlungen zumindest anfangs nicht einfach und eine Frage der Anpassung ist: 
„Anfangs hängten viele Menschen ihre Wäsche draußen vor den Fenstern auf [was verboten ist]. [...] Hier spielten sie Reggaeton, dort Salsa, Vallenato, es war ein schrecklicher Lärm. So war es für einige Zeit. Und sicher, während die Leute sich kennenlernten, kam es zu einigen ,Zusammenstößen“.“ (Interview, Bogotá im April 2016, Übers. d. A.)

Bewohner_innen der Wohnsiedlungen sind sich weitgehend einige darin, dass die Ursache der Konflikte in einer mangelnden Kenntnis der Regeln oder der Nichtbeachtung der Regeln des Zusammenlebens besteht und sie sehen das als ein kulturelles Problem an, als eine Folge der ,Kultur der Armut'[19]. Auch die Fähigkeit, sich an die neue Lebensweise anzupassen, wird als Kriterium genutzt, um zwischen ,guten' und ,schlechten' Nachbar_innen zu unterscheiden, wobei dieser Differenzierung ein Klassendenken zugrunde liegt, das Wohnungseigentum in Siedlungen mit der Mittelklasse assoziiert. Das heißt, in einer geschlossenen Wohnsiedlung zu leben, bedeutet Teil der Mittelklasse zu sein, und deshalb sollten sich die Bewohner_innen den Regeln entsprechend benehmen. Wer dies nicht tut, ist dann eben noch zu sehr der Kultur der informellen Siedlung verhaftet.

Ein zusätzliches Argument für die strikten Regeln ist die Wertsteigerung des Eigentums. Die Verwalterin eines am südwestlichen Rand Bogotás gelegenen Wohnviertels drückte es so aus:

„Ich betone der Gemeinschaft gegenüber immer wieder, dass ihre Wohnung sich nicht wegen der Fliesen [im Bad] oder der Küche, die sie eingebaut haben, valorisiert. Eine Wohnung gewinnt mehr an Wert durch das gute Zusammenleben in der Siedlung, als durch all das, was sie privat in ihre Wohnung investiert haben.“ (Interview, Bogotá im März 2016, Übers. d. A.)

Damit kommen wir zurück zum Aspekt der Finanzialisierung und der ökonomischen Funktion oder Bedeutung von Wohnraum. Die Wertsteigerung ihres Eigentums ist für die Menschen, die kürzlich aus prekären Lebensumständen aufgestiegenen sind und jetzt in den Sozialwohnungsbausiedlungen leben, extrem wichtig. Die Wohnung wird (auch) als Investition gesehen. Umso

Abb. 7 und 8

Verbotsschilder und öffentliche Sanktion an Postfächern (Fotos: Autorinnen) entscheidender ist daher aber auch, dass die Bewohner_innen Zugang zu Erwerbstätigkeiten haben, die es ihnen erlauben ihre Kredite abzuzahlen und sonstigen finanziellen Verpflichtungen (z. B. Verwaltungs- und Instandhaltungskosten) nachzukommen. Aber gerade in dieser Beziehung erweisen sich die vielen Verbote und Regeln in den Wohnsiedlungen als hinderlich.
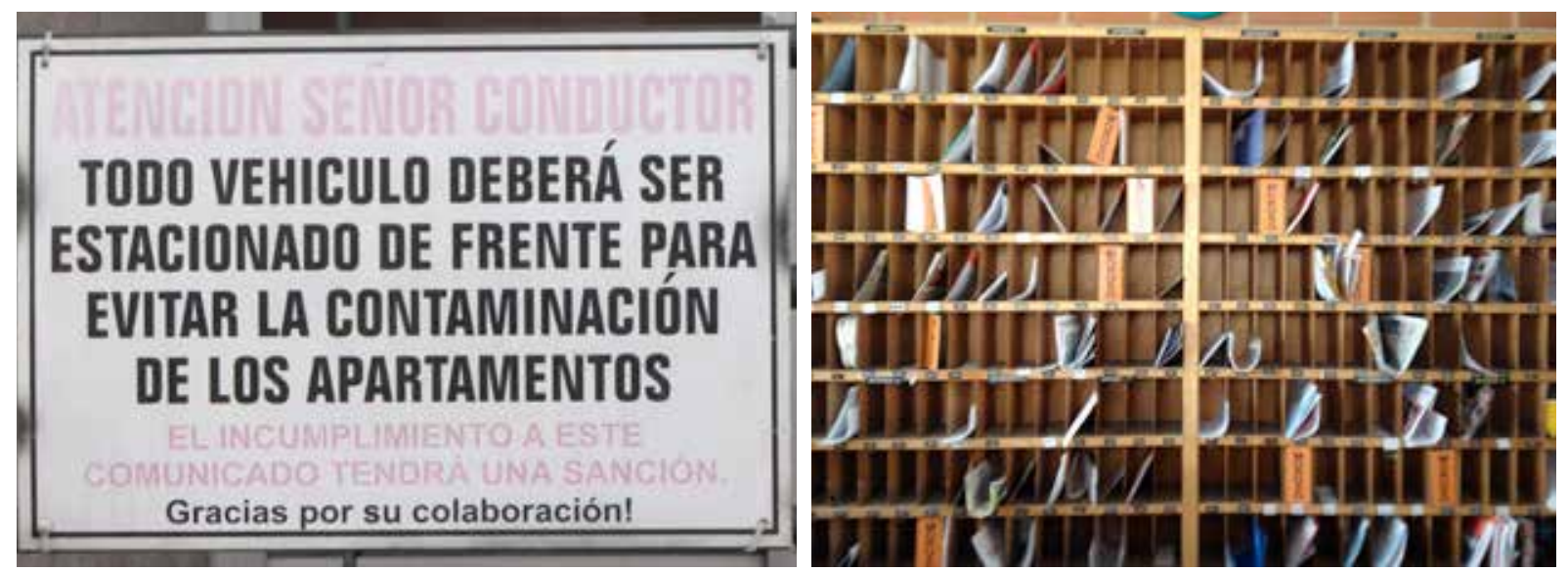
So sind etwa gewerbliche Tätigkeiten innerhalb der Siedlungen verboten. Die Baufirmen, die zumindest im ersten Jahr die Verwaltung übernehmen, begründen dies mit angeblichen gesetzlichen Vorlagen, tatsächlich handelt es sich aber um eine Auslegung eines unklaren Rechtsbestandes. Es liegt daher nahe, das Verbot gewerblicher Tätigkeiten und anderer Praktiken als Wunsch zu interpretieren, eine bestimmte Wohnästhetik und moral zu fördern, die sich durch extreme Ordnung und Regelbefolgung, Standardisierung und Anonymität auszeichnet.

Die Unterdrückung kommerzieller Tätigkeiten hat jedoch mindestens zwei negative Auswirkungen auf das Wohlergehen der Bewohner_innen von Sozialwohnungsbausiedlungen an den Rändern der Stadt. Erstens sind eine große Anzahl von Kolumbiens Niedrigverdiener_innen auf alternative oder informelle Einkommensquellen angewiesen, entweder weil sie keine feste Anstellung haben[20], oder aber, weil das Einkommen aus einer solchen Erwerbstätigkeit nicht ausreicht. In den illegalen oder informellen Siedlungen, in denen die Bewohner_innen zuvor mehrheitlich lebten, ist es üblich, in der Wohnstätte Geschäfte und sogar Unternehmen zu betreiben. Tagsüber verwandelt sich das Wohnzimmer in einen Kiosk, eine Spielgruppe für Kleinkinder, einen Friseursalon, oder es werden Zimmer vermietet. Das Wegfallen dieser Einkommensquellen prekarisiert die Bewohner_innen der Siedlungen.

Zweitens sind Kleingewerbe wichtige Orte sozialer Interaktion, die das Zusammenleben und die Solidarität unter den Bewohner_innen positiv beeinflussen kann, wie wir in einer der von uns untersuchten Wohnsiedlungen deutlich beobachten konnten. Mit Unterstützung der Verwalterin hatten sich die Bewohner_innen hier über das Verbot kommerzieller Tätigkeiten hinweggesetzt und mehrere Eigentümer_innen betrieben im Erdgeschoss ihrer Reihenhäuser kleine Kioske, wo sie zum Beispiel Erfrischungsgetränke und Snacks, Telefonkarten und Ähnliches verkauften. Diese Gewerbe hatten einen positiven Einfluss auf das Zusammenleben: Nicht nur kannte sich prozentual der größte Teil der Bewohner_innen in dieser Wohnsiedlung beim Namen und hatte die Wohnung ihrer Nachbar_innen von innen gesehen[21], sondern sie hatten auch großes Vertrauen in ihre Nachbar_innen. Als Beweis für Letzteres verwiesen Bewohner_innen darauf, dass in den Geschäften angeschrieben wurde und sie sich gegenseitig Geld liehen. Dieses soziale Miteinander wird jedoch von den Baufirmen nicht als positiv oder förderlich angesehen. So verglich ein hochrangiger Mitarbeiter das angeregte Leben in der beschriebenen Wohnsiedlung verächtlich mit einem ,Dorfjahrmarkt‘ (feria del pueblo).

Baufirmen und Regierung, aber auch viele Bewohner_innen, stellen sich die ideale Wohnsiedlung eher so vor wie die im nordwestlichen Randgebiet Bogotás liegende, neuere Siedlung „Villa Real“ (siehe Abb. 2). Villa Real war zur Zeit unserer Forschung in der Tat in einem vorbildlichen Zustand; alles war aufgeräumt und sauber, die Autos ordentlich auf den vorgesehenen Stellplätzen geparkt. Zwischen den Gebäuden gab es einige wenige gepflegte Grünflächen, Spielplätze und Sitzplätze. Allerdings fiel auf, dass sich praktisch niemand im öffentlichen Raum aufhielt. In unseren Befragungen und Interviews wurde deutlich, dass es keine oder nur minimale Interaktion zwischen den Bewohner_innen gab. Sie kannten einander nicht beim Namen und hatten sich in der Regel auch noch nie gegenseitig besucht. Darüber hinaus 
drückten die Bewohner_innen auch kein Interesse aus, ihre Nachbar_innen kennenzulernen oder etwas mit ihnen zu unternehmen. Es schien praktisch keine Kommunikation zwischen den Bewohner_innen zu geben; jegliche Vereinbarungen, Aktivitäten oder Probleme unter den Bewohner_innen wurden ausschließlich über den Verwalter erörtert oder vermittelt. Zudem beschränkten Regeln und strikte Sanktionen praktisch alle gemeinschaftlichen Aktivitäten und Interaktionen zwischen den Nachbar_innen.

Faszinierend ist, dass einige der Bewohner_innen diese Ästhetik und Moral kritiklos übernehmen, obwohl dies nicht unbedingt in ihrem besten Interesse ist. Dies liegt daran, dass das Leben in geschlossenen Wohnsiedlungen, die denen der Mittel- und Oberschicht ähneln, den Menschen das Gefühl des sozialen Aufstiegs vermittelt. Egal wie schwierig es ist, die Kreditraten zu bezahlen und als Familie auf dem begrenzten Raum und weitab an den Rändern der Stadt zu leben - für die unteren Einkommensklassen bedeutet der Besitz einer solchen Wohneinheit, dass sie reguläre Stadtbewohner_innen geworden sind (Hurtado Tarazona 2018). Ob dies wirklich so ist, bleibt jedoch fraglich, da sich an den generellen strukturellen Ungleichheiten und kulturellen Vorurteilen in Kolumbien und Bogotá nichts geändert hat. Sozialwohnungsbaubewohner_innen leben im,territorio de los comanches und werden weiterhin mit dem geographischen und sozialen ,Rand' der (urbanen) Gesellschaft assoziiert.

\section{Schlussbetrachtungen}

Der privat finanzierte soziale Wohnungsbau Kolumbiens hat weitgreifende Auswirkungen und Folgen sowohl für die Menschen persönlich als auch strukturell für die Stadt. Obwohl eine beträchtliche Anzahl der informellen Siedlungsbewohner_innen und Niedrigverdiener_innen Bogotás vom vermehrten und subventionierten Wohnungsbau profitiert haben, wird der soziale Aspekt der Wohnungsbaupolitik dadurch eingeschränkt, dass dieser Menschen in formellen Arbeitsverhältnissen vorbehalten ist und somit fast die Hälfte der kolumbianischen Bevölkerung ausgeschlossen bleibt. Aber selbst für diejenigen, die sich in die Sozialwohnungsbausiedlungen einkaufen können, ist die neue Lebensform nicht unbedingt förderlich für eine nachhaltige soziale Mobilität oder auch nur vereinbar damit. In diesem Artikel haben wir gezeigt, dass das maßgeblich damit zusammenhängt, dass die Art und Weise, wie die neuen Wohnsiedlungen gebaut und reguliert sind, den markwirtschaftlichen Interessen der Regierung und der privaten Baufirmen entspricht, die eine,unzivilisierte 'Bevölkerung erziehen wollen, die zuvor in informellen Siedlungen gewohnt hat. Anstatt Orte und Möglichkeiten der sozialen Interaktion und des Austausches zu schaffen, die das Vertrauen und die Solidarität in den Siedlungen fördern könnten, geht es den Baufirmen vornehmlich darum, neuen Hausbesitzer_innen beizubringen, wie man in Wohnsiedlungen lebt, das heißt, Regeln der Sicherheit und Hygiene sowie finanzielle Verpflichtungen einzuhalten.

Während die Nationalregierung den Aufstieg Kolumbiens in ein ,Land der Mittelklasse‘ feiert, die maßgeblich mit dem Hausbesitzer_innentum verbunden wird, bleibt fraglich, wie nachhaltig diese Transformationen sind. Der offizielle Diskurs verschleiert zum einen, dass die Sozialpolitik Ressourcen von 
den Bedürftigsten auf diejenigen mit größerer Wirtschafts- beziehungsweise Kaufkraft verlagert. Zum anderen besteht die Gefahr, dass diejenigen, die sich durch die Sozialwohnungsbaupolitik den ,Traum vom Wohnungseigentum“ in den neuen Wohnvierteln erfüllt haben, weiterhin marginalisiert bleiben. Ihnen fehlen über die angesprochenen Aspekte hinaus der Zugang zu gesicherten Arbeitsplätzen oder Einkommensquellen, zu Bildung, sozialen Dienstleistungen sowie Transportmöglichkeiten und die Einbindung in den urbanen Raum beziehungsweise diese werden ihnen verweigert (weiterführend Hurtado Tarazona et al. o. J.). Ohne tiefgreifende strukturelle gesellschaftliche Veränderungen der kolumbianischen Klassengesellschaft ist der langfristige Erfolg der gegenwärtigen Sozialwohnungsbaupolitik zweifelhaft und es besteht die Gefahr, dass sich die riesigen, sterilen Siedlungen an den Rändern der Stadt in Marginalsiedlungen verwandeln.

\section{Endnoten}

[1] In Kolumbien werden informelle Siedlungen in ,Piraten-“ (barrio pirata) und ,Invasionssiedlungen' (barrio de invasion) unterschieden. Bei den ersteren handelt es sich weitestgehend um periphere Gebiete, in denen Bewohner_innen Grundstücke käuflich erworben haben, wobei die Rechtslage dieser Transaktion nicht immer ganz eindeutig ist. Invasionssiedlungen können auch in zentraleren Gegenden der Stadt liegen, da es sich hierbei um Inbesitznahme öffentlichen oder ungenutzten Raumes handelt. Die Regierung Kolumbiens hat die Existenz informeller Siedlungen als unausweichlich akzeptiert und erkennt in der Regel beide Formen nach einer gewissen Zeit rechtlich an, wobei dies bei Piratensiedlungen zumeist schneller passiert, da die Rechtslage einfacher ist und eine Kauftransaktion zugrunde liegt. Beide Arten informeller Siedlungen in Kolumbien sind heute weitgehend mit Wasser und Elektrizität versorgt. Dies steht im Gegensatz zu illegalen Siedlungen, d. h. solchen, die nicht vom Staat anerkannt sind und die in Abständen geräumt werden (Camargo Sierra/Hurtado Tarazona 2012).

[2] 2018 waren laut dem Nationalen Statistischen Amt (DANE 2018) 48 Prozent der Bevölkerung Kolumbiens im informellen Sektor tätig.

[3] Die diesem Artikel zugrunde liegende Studie entwickelte sich aus einer Konsultation zur Evaluierung des Programmes zur Schaffung von ,Sozialkapital‘ seitens einer Konstruktionsfirma. Das Untersuchungsteam, bestehend aus vier Stadtforscher_innen und zehn Student_innen, analysierte Statistiken und Studien von 181 Sozialwohnungsbauvierteln. In neun dieser Wohnsiedlungen stellten wir vertiefende Studien an, einschließlich einer Umfrage mit 200 Personen, semi-strukturierte Interviews mit Bewohner_innen und Verwaltungspersonal sowie ethnografischen Beobachtungen.

[4] Im Falle der Selbstbauprojekte wurde der Kauf von Landparzellen für die progressive Siedlungsentwicklung für die untersten Einkommensschichten subventioniert.

[5] UPAC steht für „Einheit konstanter Kaufkraft“. Dieses System hatte seinen Ursprung in Brasilien und wurde 1972 während der Präsidentschaft von Misael Pastrana Borrero in Kolumbien eingeführt, um die Kaufkraft der Währung aufrecht zu erhalten und der Bevölkerung langfristige Hypothekarkredite zum Kauf von Wohnraum zu ermöglichen. Hypothekarkredite für Hauskäufe und Sparkonten im UPAC passten ihren Wert dem Inflationsindex an; das heißt, Zinsen würden inflationsabhängig anerkannt oder erhoben. Dadurch wurde sichergestellt, dass das Geld seine Kaufkraft behielt.

[6] Interessanterweise beinhaltete das Gesetz zunächst verschiedene Modalitäten zur Verwendung der Subventionen, wie z. B. den Kauf fertiger Wohneinheiten, den Erwerb von Grundstücken, den progressiven Wohnungsbau oder aber den Kauf von Materialien zur Verbesserung existierenden Wohnraumes oder den Erwerb von gebrauchten Wohnungen. Schlussendlich wurde jedoch der größte Teil des Geldes zum Kauf von neuen Wohneinheiten verwendet (Anzellini et al. 2012). 
[7] Die Mehrheit der Banken sind zudem mit den Konstruktionsfirmen in großen Konglomeraten verbunden, zu denen außerdem Unternehmen gehören, die Baumaterialien wie z. B. Zement produzieren. Faktisch kontrollieren heutzutage einige wenige einflussreiche Familienunternehmen den gesamten Bausektor.

[8] Siehe http://www.metrovivienda.gov.co (letzter Zugriff am 15.12.2018).

[9] Artikel 79 des Gesetzes Nummer 1151 aus dem Jahr 2007.

[10] Gesetz Nummer 1469 aus dem Jahr 2011.

[11] Obwohl internationale Ideen und Ziele wie Nachhaltigkeit, Risikomanagement, Wohnqualität und Umgebung, Verkehrsverbindungen, Ausstattung und öffentliche Dienstleitungen in die Politik und in die sozialen Wohnungsbauprojekte in Kolumbien einfließen, ist das Hauptmotiv räumlicher Interventionen noch immer das Ziel der Modernisierung. Zeiderman (2016) argumentiert, dass dies damit zu tun hat, dass in Kolumbien die wirtschaftliche Modernisierung immer eher ein Versprechen denn Realität war. „Der Sozialstaat ist niemals vollständig realisiert worden und der Liberalismus ein fortdauerndes Projekt mit limitiertem Erfolg geblieben.“ (2016: 129, Übers. d. A.) Wenn wir über ,sozialen Wohnungsbau' in Kolumbien sprechen, ist dies daher etwas anderes als in Ländern mit einer anderen Entwicklungsgeschichte.

[12] ,Finanzialisierung، beschreibt einen wirtschaftlichen Prozess, bei dem der Austausch durch die Vermittlung von Finanzinstrumenten erleichtert wird. Die Finanzialisierung kann es ermöglichen, dass reale Güter, Dienstleistungen und Risiken leicht gegen Geld austauschbar sind, und es so den Menschen erleichtern, ihre Vermögenswerte und Einkommensströme zu rationalisieren (Engel 2015).

[13] Die Einteilung des Stadtgebietes nach Schichten beruht auf der Beurteilung der Qualität der bestehenden Gebäude und der strukturellen Umgebung, was für repräsentativ für die Zahlungsfähigkeit der Bewohner_innen gehalten wird (Gesetz Nr. 142 von 1994).

[14] Nur die mittleren Schichten 3 und 4 zahlen den wirklichen Preis der Leistungen (Gesetz Nr. 142 von 1994).

[15] Die Comanchen sind ein nordamerikanisches indigenes Volk. Gespräch mit Führungskräften von Bauunternehmen, April 2016.

[16] Von 2012 auf 2015 hat sich die Zahl der Mitglieder in kolumbianischen Familien von durchschnittlich 4,3 auf 3,5 Mitglieder verringert (Profamilia 2015). Die Haushaltsgröße unterscheidet sich jedoch nach Schichten, mit mehr Familienmitgliedern in niedrigeren Schichten: 4,8 in Schicht 1 gegenüber 3,8 in Schicht 5 und 3,4 in Schicht 6, den beiden höchsten (Centro Nacional de Consultoría 2012).

[17] Alle angeführten Direktzitate stammen aus auf Tonband aufgenommenen Interviews mit Bewohner_innen und Verwalter_innen der Sozialwohnungsbausiedlungen, die Gegenstand unsers Forschungsprojektes waren.

[18] Die Bestimmungen basieren auf nationalen Gesetzen, werden aber von den Bewohner_innen selbst endgültig im Handbuch der Eigentümer_innen festgelegt. In unserer Untersuchung stellten wir fest, dass diese Interpretationen der Regeln i. d. R. strenger sind als die Vorschriften selbst.

[19] Oskar Lewis prägte 1959 den Begriff ,Kultur der Armut‘ (culture of poverty), um zu erklären, warum Menschen arm sind. Er beschrieb für arme Menschen angeblich charakteristisches Verhalten und Werte, die sich von Generation zu Generation ,vererben ' und sie von der Mittelschicht unterscheiden. Das Konzept wird heute weitgehend abgelehnt, da es strukturelle Ursachen von Armut übersieht und so letztendlich den Armen selbst die Schuld an ihrer Situation zuschreibt (siehe Davis 2012).

[20] Obwohl der Zugang zu den staatlich subventionierten Krediten eine feste Anstellung voraussetzt, erzählten uns mehrere Sozialwohnungsbesitzer_innen, dass sie,Hilfe‘ gehabt hätten, um diesem Kriterium zu entsprechen, und tatsächlich im informellen Sektor arbeiteten.

[21] Dies wird als Indikator größerer Vertrautheit angesehen als lediglich den Namen eines_r Nachbar_in zu kennen. 


\section{Autor_innen}

Friederike Fleischer ist Kulturanthropologin und arbeitet im Bereich der Stadtforschung zu sozialer und wirtschaftlicher Ungleichheit in China und Kolumbien.

f.fleischer406@uniandes.edu.co

Adriana Hurtado Tarazona ist Kulturanthropologin und Master in Stadtplanung und Regionalentwicklungsmanagement. Sie arbeitet zu städtischer Ungleichheit.

a.hurtado10@uniandes.edu.co

Maria Jose Alvarez Rivadulla ist Soziologin und analysiert soziale Ungleichheit und verwandte Themen im Bereich der Stadtforschung.

mj.alvarez@uniandes.edu.co

\section{Literatur}

Aalbers, Manuel B. (2017): The Variegated Financialization of Housing. In: International Journal of Urban and Regional Research 41/4, 542-554.

Anzellini, Stefano / Escallón, Clemencia / Herrera, Nathalie (2012): La vivienda digna en Colombia: una deuda pendiente. In: Marcela Ángel / Maria Cecilia O’Byrne (Hg.), Casa+Casa+casa=¿Ciudad? Germán Samper: Una investigación en vivienda. Bogotá: Universidad de los Andes, 252-273.

Bermúdez, Pedro/ Gaviria, Eugenia /Salcedo, Juana (2012): Panorama de la vivienda y la ciudad en el Siglo XX. In: Marcela Ángel / Maria Cecilia O’Byrne (Hg.), Casa+Casa+casa=¿Ciudad? Germán Samper: Una investigación en vivienda. Bogotá: Universidad de los Andes, 56-62.

Camargo Sierra, Angelica Patricia/ Hurtado Tarazona, Adriana (2012): Informalidad del siglo XXI. Características de la oferta informal de suelo y vivienda en Bogotá durante la primera década del siglo XXI. In: Territorios 27, 71-104.

Castro, Fatimah Williams (2013): Afro-Colombians and the Cosmopolitan City. New Negotiations of Race and Space in Bogotá, Colombia. In: Latin American Perspectives 189 /40,2, 105-117.

Centro Nacional de Consultoría (2012): ¿Como es la nueva familia colombiana? Resultados de la gran encuesta del Centro Nacional de Consultoría en las principales ciudades del país. Revista Credencial, Mai 9. http://www.revistacredencial.com/credencial/noticia/ actualidad/como-es-la-nueva-familia-colombiana (letzter Zugriff am 15.12.2018).

Cuervo, Isabel P. (2013): The different meanings of home for residents and professionals in the planning and design of social housing in Colombia. Dissertation. New York: City University of New York.

Cuervo, Nicolás / Jaramillo, Samuel (2009): Dos décadas de política de vivienda en Bogotá apostando por el mercado. Documento CEDE-Uniandes 31. Bogotá: Universidad de los Andes.

Departamento Administrativo Nacional de Estadística (DANE) (2018) Medición de empleo informal y seguridad social. Boletin Tecnico. Bogotá. https://www.dane.gov.co/files/ investigaciones/boletines/ech/ech_informalidad/bol_ech_informalidad_may18_jul18. pdf (letzter Zugriff am 20.1.2019).

Davis, Dana-Ain (2012): Culture of Poverty. Oxford Bibliographies. http://www.oxfordbibliographies.com/view/document/obo-9780199766567/obo-9780199766567-0004. xml (letzter Zugriff am 20.1.2019).

Departamento Nacional de Planeación (DNP) (2011): Bases del Plan Nacional de Desarrollo 2010-2014: Bogotá. https://colaboracion.dnp.gov.co/CDT/PND/Bases\%20PND\%20 2010-2014\%20Versión\%205\%2014-04-2011\%20completo.pdf (letzter Zugriff am 20.1.2019).

Engel, Alexander (2015): The Bang after the Boom: Understanding Financialization. In: Zeithistorische Forschungen/Studies in Contemporary History 12, 500-510.

Fleischer, Friederike / Marín, Keren (2019): Atravesando la ciudad. La movilidad y experiencia subjetiva del espacio por las empleadas domésticas en Bogotá. In: EURE 45/135, 27-47.

Forrest, Ray / Hirayama, Yosuke (2011): Neoliberalism and the Reproduction of Home Ownership. Paper presented at the International RC21 conference 2011 Amsterdam. 
http://www.rc21.org/conferences/amsterdam2011/edocs2/Session\%204/RT4-1Forrest.pdf (letzter Zugriff am 9.2.2019).

Girola, Maria Florencia (2007): Procesos de apropiación del espacio y sociabilidad vecinal en un gran conjunto urbano situado en la ciudad de Buenos Aires. In: Anthropologica 25/25, 131-155.

Hurtado Tarazona, Adriana / Alvarez Rivadulla, Maria Jose / Fleischer, Friederike (o. J.): The Normalization of Bogota Social Housing Residents. Class tensions in Third World Urban Peripheries. City \& Society. Im Ersch.

Hurtado Tarazona, Adriana (2018): Habitar Como Labor Material y Simbólica: La Construcción de Un Mundo Social En Ciudad Verde (Soacha, Colombia). Thesis zur Erlangung der Doktorwürde. Bogotá: Universidad de los Andes.

Klein, Charles / Mitchell, Sean T. / Junge, Benjamin (2018): Naming Brazil's previously poor: „New middle class“ as an economic, political, and experiential category. In: Economic Anthropology 5, 83-95.

Minvivienda (2015): Dekret Nummer 0428. http://www.minvivienda.gov.co/Decretos\%20 Vivienda/0428\%20-\%202015.pdf (letzter Zugriff am 12.9.2019).

Parias,Adriana (2012): Configuración urbanayviviendapopularen Bogotáenlasegundamitad del siglo XX. In: Marcela Ángel / Maria Cecilia O’Byrne (Hg.), Casa+Casa+casa=¿Ciudad? Germán Samper: Una investigación en vivienda. Bogotá: Universidad de los Andes, 74-87.

Pérez, Federico (2010): Laboratorios de reconstrucción urbana: Hacia una antropología de la política urbana en Colombia. In: Antipoda. Revista de Antropología y Arqueología 10, 51-84.

Portafolio (2015): „Somos un país de clase media“, Juan Manuel Santos. https://www.portafolio.co/economia/finanzas/pais-clase-media-juan-manuel-santos-40942 (letzter Zugriff am 31.7.2018).

Profamilia (2015): Encuesta Nacional de Demografia y Salud 2015. Bogotá, Colombia. http:// profamilia.org.co/docs/ENDS\%20\%20TOMO\%20I.pdf (letzter Zugriff am 13.2.2019).

Rolnik, Raquel (2013): Late Neoliberalism: The Financialization of Homeownership and Housing Rights. In: International Journal of Urban and Regional Research 37/3, 1058-1066.

Rolnik, Raquel / Rabinovich, Lidia (2014): Late Neoliberalism: the financialisation of homeownership and the housing rights of the poor. In: Aoife Nolan (Hg.), Economic and Social Rights after the Global Financial Crisis, 57-89.

Saldarriaga Roa, Alberto / Carrascal, Rodrigo (2006): La vivienda social en Colombia. Bogotá: Premio Corona.

Semana (2015): Colombia, un país de clase media. https://www.semana.com/nacion/ articulo/colombia-un-pais-de-clase-media/427747-3 (letzter Zugriff am 10.9.2016).

UN-Habitat (1976): The Vancouver Declaration on Human Settlements. Vancouver: United Nations Conference on Human Settlements.

World Bank (2018): Nearly Half the World Lives on Less than \$5.50 a Day. The World Bank, Oktober 2018. https://www.worldbank.org/en/news/press-release/2018/10/17/nearlyhalf-the-world-lives-on-less-than-550-a-day (letzter Zugriff am 13.9.2019).

Zeiderman, Austin (2016): Endangered City: The Politics of Security and Risk in Bogotá. Durham/London: Duke University Press.

\section{Ghettos on the Edge of the City? Social housing policy and social inequality in Colombia}

Faced with a growing demand for affordable housing, in the 1980s the Colombian government embarked on a new, privatized housing policy designed to facilitate and promote homeownership. For this purpose, state subsidies and special mortgages for the lower income classes were created, as well as price limits and tax-exempt agreements for private construction companies. Especially since the economic crisis at the beginning of the millennium, private construction companies focus on social housing construction for profits. Since then, large-scale standardized housing estates have 
sprung up on the outskirts of Bogotá. Residents are low-income earners who previously lived in informal settlements. To access the state-subsidized mortgages, buyers must have permanent employment, which excludes half of the Colombian population. Based on multi-method collected research data in nine different social housing areas, in this article we analyze the current housing policy of Colombia and its consequences. We argue that the new policy actually gives more people access to condominiums. At the same time, we show that without further social reforms there is a risk that the isolated housing estates will turn into urban ghettos that continue to stigmatize their inhabitants. Access to education, jobs, culture and other urban resources is necessary to ensure long-term social mobility. 